\title{
Wie lässt sich die ärztliche Versorgung bewerten?
}

\author{
Valérie Gloor ${ }^{a}$, Christian Ambord ${ }^{b}$, Monique Lehky Hagen ${ }^{c}$, Luc Fornerod ${ }^{a}$, Arnaud Chiolero ${ }^{a}$ \\ a Walliser Gesundheitsobservatorium (WGO), Sitten; ${ }^{b}$ Dienststelle für Gesundheitswesen, Sitten; ${ }^{c}$ Walliser Ärztegesellschaft (VSÄG), Sitten.
}

\section{Mehr Ärzte - doch für welches Angebot?}

Zur Evaluation der ärztlichen Versorgung werden oftmals die absolute Ärztezahl und die Ärztedichte verwendet. Die Zahl der berufstätigen Ärzte hat in der Schweiz in den letzten 20 Jahren stark zugenommen: laut Statistiken der FMH von 20030 im Jahr 1990 auf 34348 im Jahr 2014 [1]. In derselben Zeitspanne hat die Ärztedichte von 3,0 auf 4,1 Ärzte pro 1000 Einwohner zugenommen. Diese Ärztedichte unterscheidet sich jedoch stark von Kanton zu Kanton. Im Jahr 2014 gab es im Kanton Zürich beispielsweise 4,9 Ärzte pro 1000 Einwohner, in der Waadt 4,6 und im Kanton Freiburg 2,6. Mit 2,8 Ärzten pro 1000 Einwohner weist das Wallis eine relativ geringe Ärztedichte auf.

Für eine Einschätzung der ärztlichen Versorgung reicht es jedoch ganz klar nicht aus, die Ärztezahl mit der Bevölkerungszahl ins Verhältnis zu setzen [2-5]. Angesichts der Faktoren, welche die Entwicklung des Versorgungsbedarfs bestimmen, müssen nämlich auch die Veränderungen auf demographischer Ebene und in Bezug auf die Ausübung des Ärzteberufs berücksichtigt werden. Die Alterung der niedergelassenen Ärzte, die Reduzierung des Arbeitspensums und die zunehmende Anzahl Frauen im Arztberuf sowie die sinkende Attraktivität des Hausarztberufs (Grundversorger) sind allesamt Faktoren, die zu berücksichtigen sind, wenn man die Entwicklung der ärztlichen Versorgung nachvollziehen will. Ausserdem sind die in der Schweiz berufstätigen Ärzte relativ alt, was bedeutet, dass kurz- oder mittelfristig viele von ihnen ihr Arbeitspensum verringern oder ihre Tätigkeit niederlegen werden. Diese Veränderungen lassen befürchten, dass es schwierig sein wird, die ärztliche Versorgung aufrechtzuerhalten [2,3]. Diese Schwierigkeit widerspiegelt sich bereits im zunehmenden Rückgriff auf im Ausland ausgebildete Ärzte (im Jahr 2014 gemäss FMH 30,5\% der Ärzte [1]).

Zusätzlich zu den Besonderheiten im Zusammenhang mit der Organisation des lokalen Gesundheitssystems und der saisonbedingten Schwankungen des Versorgungsbedarfs (z.B. im Zusammenhang mit dem Tourismus) muss daher unbedingt das Arbeitspensum der Ärzte berücksichtigt werden, um die ärztliche Versorgung kor- rekt zu evaluieren. Beispielsweise wird die ärztliche Versorgung in einer gegebenen Region sehr unterschiedlich ausfallen, ob die Ärzte nun sechs Tage die Woche oder ob dieselbe Anzahl Ärzte bloss drei Tage arbeiten. Da die Ärzte dazu tendieren, ihr Arbeitspensum zu reduzieren [6], kann eine Zunahme der Ärztezahl mit einer gleichbleibenden oder sogar abnehmenden effektiven ärztlichen Versorgung einhergehen.

Obschon die FMH Statistiken über sämtliche Ärzte in der Schweiz, die FMH-Mitglieder sind, erstellt, geben diese nur teilweise Auskunft über das Arbeitspensum und machen keinerlei Aussagen zu geplanten Änderungen der Tätigkeit. Um dem Bedarf einen Schritt voraus zu sein und geeignete Massnahmen zu ergreifen, ist es wichtig, die Situation auf Ebene der einzelnen Kantone - und sogar auf Ebene der Regionen - genau zu evaluieren. Verfügt man nämlich über abschliessende Daten zu allen Ärzten, kann man die oftmals lokalen Probleme, welche Massnahmen auf regionaler bzw. kommunaler Ebene erforderlich machen, nachvollziehen.

Im Wallis hat das Gesundheitsdepartement eine Expertenkommission "Ambulante Pflege und Grundversorgung» ernannt, um die Situation der ärztlichen Grundversorgung zu analysieren und der öffentlichen Hand diesbezügliche Empfehlungen abzugeben [7, 8]. Hierzu benötigte die Kommission Informationen über die Tätigkeit der Ärzte. Infolge der verschiedenen Änderungen der Reglementierung über die Einschränkung der Zulassung von Leistungserbringern zur Tätigkeit zu Lasten der obligatorischen Krankenpflegeversicherung [5] ist es für die Walliser Dienststelle für Gesundheitswesen (DGW) unentbehrlich geworden, über qualitativ hochstehende und umfassende Informationen über die Tätigkeit der Ärzte zu verfügen. Eine gute Zusammenarbeit mit der Walliser Ärztegesellschaft (VSÄG) wurde als grundlegend erachtet, weshalb diese eng in die Überlegungen miteinbezogen wurde. Das Walliser Gesundheitsobservatorium (WGO) wurde beauftragt, in enger Zusammenarbeit mit der DGW und der VSÄG eine Umfrage zur Tätigkeit der Ärzte durchzuführen, deren Ergebnisse nachstehend aufgezeigt werden. 
Tabelle 1: Anzahl Vollzeitstellen (VZS) der ärztlichen Grundversorger und Dichte pro Tausend Einwohner nach verfassungsmässiger Region, Wallis, 2014. Eine VZS entspricht 10 Halbtagen Tätigkeit eines Arztes (Quelle: WGO [10]).

\begin{tabular}{|c|c|c|c|c|}
\hline & Oberwallis & Mittelwallis & Unterwallis & Total \\
\hline AnzahI VZS & 60,1 & 114,8 & 73,1 & 248,0 \\
\hline Anzahl Grundversorger & 68 & 139 & 89 & 296 \\
\hline VZS-Dichte/1000 Einwohner & 0,7 & 0,9 & 0,6 & 0,8 \\
\hline Städtischer Raum & 1,0 & 1,3 & 1,0 & 1,1 \\
\hline
\end{tabular}

\section{Methodik}

Mit dieser von der Walliser Dienststelle für Gesundheitswesen (DGW) und vom Walliser Gesundheitsobservatorium (WGO; www.ovs.ch) [9] in Zusammenarbeit mit der Walliser Ärztegesellschaft (VSÄG) durchgeführten Erhebung sollte die Tätigkeit aller niedergelassenen oder in einem Spital tätigen Ärzte, die im Jahr 2014 über eine Berufsausübungsbewilligung verfügten, dokumentiert werden. Die Assistenzärzte und Oberärzte wurden in die Erhebung nicht miteinbezogen.

Die DGW und das WGO hatten einen zweisprachigen Fragebogen ausgearbeitet, der vor dem Versand dem VSÄG unterbreitet wurde. Die Umfrage wurde vom 10. Februar 2014 bis zum 19. August 2014 durchgeführt. Jeder zur Teilnahme aufgeforderte Arzt erhielt von der DGW eine E-Mail, begleitet von einem Unterstützungsschreiben der VSÄG, in welcher er gebeten wurde, den Online-Fragebogen auszufüllen. Der Zugang zum Fragebogen wurde in einer persönlichen E-Mail zusammen mit einem Zugangskonto und einem persönlichen Login-Code bekanntgegeben. Reagierte ein Arzt auf diese E-Mail nicht, wurde ihm per Mail eine Erinnerung geschickt. Einigen Ärzten wurde ein Fragebogen in

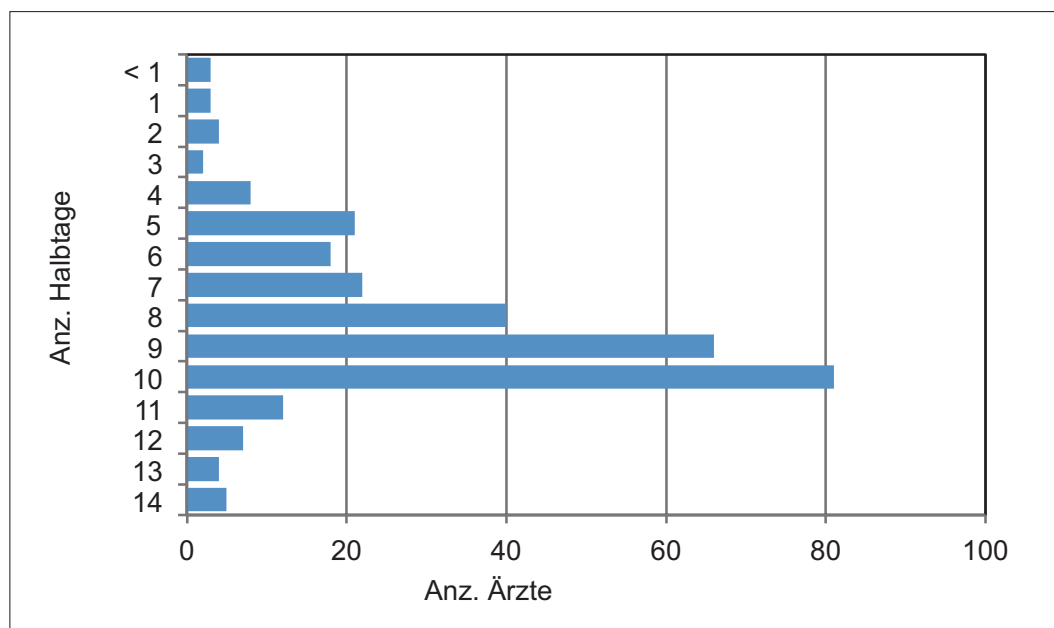

Abbildung 1: Ärztliche Grundversorger nach Anzahl Halbtage Tätigkeit pro Woche, Wallis, 2014 (Quelle: WGO [10]).
Papierformat zugeschickt. Die Ärzte, die nicht geantwortet hatten, wurden angerufen, um den Grund für die ausbleibende Teilnahme zu erfahren oder um sie den Fragebogen telefonisch beantworten zu lassen.

Die Daten wurden vom WGO anonym behandelt. Die vorläufigen Ergebnisse wurden der VSÄG anlässlich einer gemeinsamen Diskussionssitzung, zu der sämtliche Mitglieder eingeladen wurden, präsentiert. Der Bericht über diese Erhebung ist auf Deutsch und Französisch auf der Website des WGO (www.ovs.ch) verfügbar [10].

\section{Ergebnisse}

\section{Ergebnisse für alle teilnehmenden Ärzte}

Von den 898 kontaktierten Ärzten mit einer Berufsausübungsbewilligung im Wallis wurden 44 ausserkantonal tätige Ärzte ausgeschlossen und 56 Ärzte hatten den Fragebogen nicht ausgefüllt (Teilnahmeverweigerung, Abwesenheit, Übertritt in den Ruhestand). Die Ergebnisse beziehen sich folglich auf 798 Ärzte, was einer Rücklaufquote von 93\% entspricht.

Das Durchschnittsalter der Ärzte liegt bei 53,0 Jahren (Oberwallis: 53,8 Jahre, Mittelwallis: 52,7, Unterwallis: 53,1). Die Haupttätigkeit von 39\% der Ärzte steht mit einem Facharzttitel FMH in medizinischer Grundversorgung (Allgemeine Innere Medizin, Kinder- und Jugendmedizin, Praktischer Arzt) im Zusammenhang, bei $61 \%$ ist es eine Tätigkeit im Zusammenhang mit einem anderen Facharzttitel FMH. Die befragten Ärzte arbeiten durchschnittlich 7,8 Halbtage pro Woche (Männer: 8,2, Frauen: 7,1). Setzt man eine Vollzeitstelle (VZS) mit 10 Halbtagen Tätigkeit eines Arztes gleich, üben diese 798 Ärzte global gesehen eine Tätigkeit von 626 VZS aus.

\section{Ergebnisse für die Grundversorger}

Als Grundversorger (N = 296; 37\% aller Ärzte) gelten im Rahmen dieser Erhebung die Ärzte, die angegeben haben, als Hausärzte tätig zu sein (ärztliche Grundversorgung anzubieten), und die nicht Kaderärzte in einem Spital sind. Sie haben entweder einen Facharzttitel in 
Allgemeiner Innerer Medizin, Kinder- und Jugendmedizin oder als Praktischer Arzt ( $N=264$, 89\% der Grundversorger), oder einen vergleichbaren Facharzttitel (Rheumatologie, Kardiologie oder Angiologie, Endokrinologie/Diabetologie, Pneumologie, Gastroenterologie, Gynäkologie und Geburtshilfe, Allergologie und klinische Immunologie, Physikalische Medizin und Rehabilitation; $\mathrm{N}=32$, 11\% der Grundversorger). Ihr Durchschnittsalter liegt bei 55,0 Jahren. Sie arbeiten durchschnittlich 8,4 Halbtage pro Woche (Abbildung 1 und Tabelle 1).

Im Durchschnitt arbeiten die Grundversorger einen Halbtag mehr als die anderen Ärzte (8,4 vs. 7,4). Global gesehen entspricht ihre Tätigkeit 248 VZS (Tabelle 1) und 90\% ihrer Tätigkeit ist der ärztlichen Grundversorgung gewidmet (Tabelle 2). 11\% der Grundversorger $(\mathrm{N}=32)$ üben hauptsächlich eine andere Fachdisziplin aus und 53\% ihrer Tätigkeit ist der ärztlichen Grundversorgung (Hausarztmedizin) gewidmet.

Die Grundversorgerdichte in VZS liegt bei 0,8 VZS pro 1000 Einwohner. Zwischen den einzelnen Regionen gibt es grosse Unterschiede: 0,7 VZS pro 1000 Einwohner im Oberwallis, 0,9 im Mittelwallis und 0,6 im Unterwallis. In jeder der Regionen ist die Ärztedichte im ländlichen Raum deutlich geringer als im städtischen Raum (Tabelle 2).

In den nächsten fünf Jahren ist mit einer Abnahme von 25 VZS im Vergleich zu den im Jahr 2014 praktizierenden Ärzten zu rechnen (Tabelle 3). Diese Abnahme an VZS bei den Grundversorgern macht $61 \%$ der gesamten Abnahme der ärztlichen Versorgung (alle Ärzte zu- sammen) aus, die in den nächsten fünf Jahren zu erwarten ist.

\section{Diskussion}

Da es nur wenig verfügbare Daten gibt, ist es schwierig, das Arbeitspensum der Ärzte zu dokumentieren [1-6]. Die FMH-Statistiken sind nützlich, doch müssen sie unbedingt durch Ad-hoc-Erhebungen ergänzt werden, um das Arbeitspensum und geplante Änderungen der Tätigkeit zu berücksichtigen, und insbesondere um über gültige Daten auf regionaler Ebene zu verfügen. Eine Statistik, die lediglich einen kantonalen Überblick vermittelt, ist auf regionaler Ebene nämlich nicht direkt verwendbar. Diese Erhebung zeigt, dass die Ärztedichte (Grundversorger) im städtischen Raum doppelt so hoch sein kann wie im ländlichen Raum.

Es ist auch wichtig, über Informationen zu geplanten Änderungen der Tätigkeit zu verfügen. Tatsächlich ist es schwierig, sich einzig auf das Alter der Ärzte zu stützen, um eine Reduzierung bzw. Aufgabe ihrer Tätigkeit vorherzusagen, da ein grosser Teil der Ärzte nicht im Alter von 65 Jahren in den Ruhestand tritt. Ausserdem ist es wichtig, die effektive Tätigkeit, und nicht bloss den FMH-Titel, zu erfassen. Diese Erhebung zeigt nämlich, dass mehr als 10\% der Ärzte, welche Leistungen der ärztlichen Grundversorgung erbringen, über einen Facharzttitel verfügen, der üblicherweise nicht zu den Grundversorgertiteln gezählt wird.

Ein Schlüsselelement für den Erfolg dieser Erhebung war die ausgezeichnete Zusammenarbeit zwischen dem

Tabelle 2: Tätigkeit insgesamt und Tätigkeit der ärztlichen Grundversorgung (hausärztliche Tätigkeit) der Grundversorger nach FMH-Titel und nach verfassungsmässiger Region, Wallis, 2014 (Quelle: WGO [10]).

\begin{tabular}{|c|c|c|c|c|c|}
\hline & & Oberwallis & Mittelwallis & Unterwallis & Total \\
\hline \multirow{4}{*}{$\begin{array}{l}\text { Grundversorger - unabhängig } \\
\text { ihres FMH-Titels }\end{array}$} & Anzahl Ärzte & 68 & 139 & 89 & 296 \\
\hline & Anzahl Halbtage total & 8,8 & 8,3 & 8,2 & 8,4 \\
\hline & $\begin{array}{l}\text { Anzahl Halbtage, die der ärztlichen } \\
\text { Grundversorgung gewidmet sind }\end{array}$ & 8,1 & 7,3 & 7,5 & 7,5 \\
\hline & $\begin{array}{l}\text { Anteil Tätigkeit, die der ärztlichen } \\
\text { Grundversorgung gewidmet ist }\end{array}$ & $91 \%$ & $89 \%$ & $91 \%$ & $90 \%$ \\
\hline \multirow{4}{*}{$\begin{array}{l}\text { A) Mit FMH-Titel in ärztlicher } \\
\text { Grundversorgung }\end{array}$} & Anzahl Ärzte & 60 & 125 & 79 & 264 \\
\hline & Anzahl Halbtage total & 8,7 & 8,2 & 8,2 & 8,3 \\
\hline & $\begin{array}{l}\text { Anzahl Halbtage, die der ärztlichen } \\
\text { Grundversorgung gewidmet sind }\end{array}$ & 8,4 & 7,7 & 7,7 & 7,9 \\
\hline & $\begin{array}{l}\text { Anteil Tätigkeit, die der ärztlichen } \\
\text { Grundversorgung gewidmet ist }\end{array}$ & $96 \%$ & $94 \%$ & $95 \%$ & $95 \%$ \\
\hline \multirow[t]{4}{*}{ B) Mit anderen FMH-Titeln } & Anzahl Ärzte & 8 & 14 & 10 & 32 \\
\hline & Anzahl Halbtage total & 9,8 & 8,9 & 8,6 & 9,0 \\
\hline & $\begin{array}{l}\text { Anzahl Halbtage, die der ärztlichen } \\
\text { Grundversorgung gewidmet sind }\end{array}$ & 5,9 & 3,8 & 5,3 & 4,8 \\
\hline & $\begin{array}{l}\text { Anteil Tätigkeit, die der ärztlichen } \\
\text { Grundversorgung gewidmet ist }\end{array}$ & $60 \%$ & $42 \%$ & $62 \%$ & $53 \%$ \\
\hline
\end{tabular}


Tabelle 3: Ärztliche Grundversorger nach geplanter Änderung des Arbeitspensums in den nächsten fünf Jahren und nach verfassungsmässiger Region, Wallis, 2014 (Quelle: WGO [10]).

\begin{tabular}{|c|c|c|c|c|c|}
\hline $\begin{array}{l}\text { Geplante Änderung des } \\
\text { Arbeitspensums in den nächsten } \\
5 \text { Jahren }\end{array}$ & Oberwallis & Mittelwallis & Unterwallis & Total & Prozent \\
\hline Ja, Erhöhung & 3 & 4 & 9 & 16 & $5 \%$ \\
\hline Ja, Reduzierung & 16 & 52 & 18 & 86 & $29 \%$ \\
\hline Weiss nicht & 11 & 23 & 12 & 46 & $16 \%$ \\
\hline
\end{tabular}

WGO, der DGW und der VSÄG. Die DGW und die VSÄG hatten beide ihren Bedarf nach gültigen Daten zu diesem Thema geäussert und beschlossen, die Durchführung der Erhebung zu unterstützen. Dies erklärt teilweise die sehr hohe Rücklaufquote. Das WGO seinerseits verfügt über die Fachkenntnisse zur Durchführung solcher Erhebungen (namentlich durch die Verwendung eines benutzerfreundlichen elektronischen Fragebogens) und zur objektiven Bearbeitung der Ergebnisse. Eine Schwäche der Erhebung liegt in der fehlenden Information zum Ärztenachwuchs. Diese Information ist schwierig zu erhalten, da die Ärzte, die sich im Wallis niederlassen, aus verschiedenen Kantonen, Spitälern und sogar aus verschiedenen Ländern kommen können. Allerdings könnte es nützlich sein, geplante Tätigkeitsaufnahmen von Assistenzärzten und Oberärzten in den Walliser Spitälern zu dokumentieren. Es wäre auch zweckmässig, systematisch die Niederlassung von Ärzten nach dem Erhalt ihrer Berufsausübungsbewilligung zu beobachten, um abzuschätzen, ob diese Ärztezahl ausreicht, um die absehbare Abnahme an VZS der nächsten fünf Jahre zu kompensieren. Schliesslich wäre es auch nützlich, die Verfügbarkeit der Ärzte zu evaluieren, beispielsweise indem ihre Kapazität zur Annahme neuer Patienten dokumentiert wird [6].

$\mathrm{Zu}$ guter Letzt muss auch daran erinnert werden, dass mit dieser Art von Erhebung wohl die ärztliche Versorgung besser abgeschätzt, jedoch keineswegs der Versorgungsbedarf der Bevölkerung beurteilt werden kann.

Korrespondenz:

Dr. Arnaud Chiolero,

PD \& MER

Chefarzt und Epidemiologe

Walliser Gesundheitsobser-

vatorium (WGO)

CH-1950 Sitten

arnaud.chiolero[at]ovs.ch
Ärzten, damit sie einem Mangel in bestimmten Bereichen vorgreifen sowie beurteilen können, in welchen Bereichen das Angebot gering oder hoch ist - all dies nach Region.

Zur Durchführung dieser Art von Erhebung ist es wichtig, die betroffenen Partner mit einzubeziehen, das heisst die Planungsinstanz (DGW), die Ärzte (VSÄG) und die Fachstelle für die Überwachung des Gesundheitswesens und die Datenanalyse (WGO). Damit kann eine geeignete Erhebung konzipiert werden, um Informationen zu sammeln, die allen Partnern zweckdienlich sind, und um konstruktiv über die Ergebnisse zu diskutieren. Zudem kann damit verhindert werden, dass einerseits mangels Kenntnissen der Gegebenheiten vor Ort falsche Auslegungen gemacht werden und andererseits die Ergebnisse subjektiv gelesen werden. In Erwartung einheitlicherer Daten über sämtliche Ärzte in der Schweiz über das Projekt MARS des BFS [11] ist es wichtig, auf Ebene der Kantone Erhebungen zur Dokumentierung der Tätigkeit der Ärzte durchzuführen. Man muss darauf achten, dass die Interpretation dieser statistischen Daten unter Einbezug der betroffenen Partner stattfindet. Die aktuellen Diskussionen rund um die Aufhebung des Zulassungsstopps von Fachärzten zeigen umso mehr, wie wichtig es ist, objektiv über das tatsächliche Angebot diskutieren zu können, indem man sich auf fundierte und verlässliche Erhebungen stützt.

Danksagung

Wir danken allen Ärzten, die an dieser Umfrage teilgenommen haben, sowie den Personen, die an der Konzipierung und Umsetzung mitgewirkt haben (DGW: Daniela Fante, administrative Mitarbeiterin, und Cédric Mizel, Verantwortlicher der juristischen Abteilung; WGO: André-Philippe Borgazzi, Verantwortlicher des Informationssystems, und Aurélie Calmeyn, Informatikerin). 


\section{Literatur}

1 Hostettler S, Kraft E. FMH-Ärztestatistik 2014. Frauenund Ausländeranteil nehmen kontinuierlich zu. Schweizerische Ärztezeitung 2015; 96: 462-9.

2 Roth $M$. Angebot und Inanspruchnahme von medizinischen Leistungen in Arztpraxen von 2005 bis 2007 (Obsan Dossier 14). Neuchâtel: Schweizerisches Gesundheitsobservatorium, 2010.

3 Vilpert S. Ärztliche Grundversorgerinnen und Grundversorger Situation in der Schweiz und im internationalen Vergleich. Analyse des International Health Policy Survey 2012 des Commonwealth Fund im Auftrag des Bundesamtes für Gesundheit (BAG), Obsan Dossier 22. Neuchâtel: Schweizerisches Gesundheitsobservatorium, 2012.

4 Favre F, Gloor V, Chiolero A. Geographische Verteilung der Hausärzte im Wallis, 2011. Walliser Gesundheitsobservatorium (WGO). Bericht zuhanden der DGW. Sitten, November 2012. www.ovs.ch/data/documents/publication/observatoire/ Geographie_medicale_Valais_12.pdf; Zugriff am 5.6.2015.

5 Roth S, Sturny I. Zulassungsstopp für Ärztinnen und Ärzte in Praxen - Entwicklung des Ärztebestands (Obsan Bulletin 4 / 2015). Neuchâtel: Schweizerisches Gesundheitsobservatorium.
6 Merçay C. Ärztliche Grundversorgerinnen und GrundversorgerSituation in der Schweiz, neuere Entwicklungen und internationaler Vergleich. Analyse des International Health Policy Survey 2015 des Commonwealth Fund im Auftrag des Bundesamtes für Gesundheit (BAG) (Obsan Dossier 50; 2015). Neuchâtel: Schweizerisches Gesundheitsobservatorium.

7 Ducret N, Ambord Ch, Pécoud A. Drohender Ärztemangel ein Kanton macht vorwärts. Schweizerische Ärztezeitung 2013; 94:738-9.

8 Pécoud A, Moret-Ducret N. Pénurie médicale: Le Valais monte au front. Revue Médicale Suisse 2014; 10.

9 Chiolero A, Paccaud F, Fornerod L. Comment faire de la surveil lance sanitaire? L'exemple de l'Observatoire valaisan de la santé en Suisse. Santé Publique 2014; 26:75-84.

10 Gloor V, Chiolero A. Walliser Gesundheitsobservatorium (WGO). Erhebung zur Tätigkeit der Ärzte im Wallis. Sitten, Juli 2015.

11 Bundesamt für Statistik. Statistiken der ambulanten Gesundheits versorgung (MARS). http://www.bfs.admin.ch/bfs/portal/de/ index/news/00/06.html; Zugriff am 5.6.2015. 